\title{
Investigating mixed-sign equilibria for nonlinear collective decision-making systems
}

Angela Fontan and Claudio Altafini

Conference article

Cite this conference article as:

Fontan, A., Altafini, C. Investigating mixed-sign equilibria for nonlinear collective decision-making systems, In Proceedings from the 2017 IEEE 56th Annual Conference on Decision and Control (CDC), December 12-15, 2017, Melbourne, Australia, 2017, pp. 781-786. ISBN: 978-1-5090-2873-3

DOI: https:// doi.org/ 10.1109/CDC.2017.8263755

Copyright: IEEE

The self-archived postprint version of this conference article is available at Linköping University Institutional Repository (DiVA):

http:// urn.kb.se/ resolve?urn=urn:nbn:se:liu:diva-145192 


\title{
Investigating mixed-sign equilibria for nonlinear collective decision-making systems*
}

\author{
Angela Fontan and Claudio Altafini ${ }^{\star}$
}

\begin{abstract}
In this paper we provide necessary conditions for the existence of multiple equilibrium points for a class of nonlinear cooperative networked systems with saturating interactions which describe models of collective decision-making. The multiple steady states of the dynamics represent the possible outcomes of a decision process, and, except for one positive and one negative, have all mixed signs. The conditions we obtain can be formulated in terms of the algebraic connectivity of the network and are inspired by Perron-Frobenius arguments. It is also shown that the mixed-sign equilibria are contained in a ball of radius given by the norm of the positive equilibrium point and centered in the origin. Numerical examples are given to illustrate the results.
\end{abstract}

\section{INTRODUCTION}

Many applications, ranging from collective decisionmaking in animal groups [1] to opinion dynamics in social communities [2], [3], from gene regulatory network [4] to neural networks [5], can be modeled as nonlinear interconnected systems, with nonlinear sigmoidal saturationlike functional forms describing the interactions between the nodes [6], [5], [7]. Such saturations avoid divergence of the dynamics but complicate the phase portrait, by inducing complex nonlinear behaviors like periodic orbits or coexistence of multiple (stable or unstable) steady states. If the saturated functional forms are also monotone, then it is possible to exclude the presence of attracting periodic orbits but not of multiple equilibria [8], [9]. Such conditions indeed occur in neural networks models, like in the so-called Hopfield [6] or Cohen-Grossberg [10] neural networks. The concept that often occurs is that of "multistability", i.e. the presence of multiple stable equilibrium points, which has been intensively investigated in the last years [11], [12], [13], [14], see also [15] for an overview. The notion this paper is focusing on is that of "multiequilibria", i.e. multiple stable and unstable equilibria for the system.

The class of nonlinear models here considered is described in [1], [16], [17], and it represents bio-inspired collective decision-making processes. The adjacency matrix of the network is nonnegative, i.e. all the interactions between the nodes are "activatory". Furthermore, it is assumed to be symmetric or diagonally symmetrizable and irreducible. The model has a Laplacian-like structure at the origin and

\footnotetext{
${ }^{*}$ Work supported in part by a grant from the Swedish Research Council (grant n. 2015-04390)

${ }^{\star}$ A. Fontan and C. Altafini are with the Division of Automatic Control, Department of Electrical Engineering, Linköping University, SE-58183 Linköping, Sweden, E-mail: \{angela.fontan, claudio.altafini\}@liu.se
}

monotone saturating nonlinearities to represent the interaction terms as in [1]. The amplitude of the interaction part is modulated by a scalar parameter, playing the role of bifurcation parameter.

According to the analysis made in [1], for small values of the bifurcation parameter the origin is globally stable, as can be easily deduced by (global) diagonal dominance. Above a critical value, the system experiences a pitchfork bifurcation: the origin becomes unstable, and two locally stable equilibria, one positive, the other negative, appear. However, the approach followed by [1] can only capture what happens in a neighborhood of the bifurcation point. The behavior of the system when the bifurcation parameter assumes bigger values is still not understood and its investigation is the task of this paper. In this way we also aim to extend the research of [16], [17], where the behavior of the system in the positive orthant is considered, to the entire $\mathbb{R}^{n}$.

As already mentioned, the notions of multiequilibria and multistability often occur in the neural networks literature. For neural networks with symmetric adjacency matrices and monotone increasing and sigmoidal nonlinearities describing the interconnections, convergence to stable equilibrium points is known since [6]. The possibility of counting the number of stable steady states is of particular interest and in [11], [12], [13] it is shown that the number of such equilibria grows exponentially with the number $n$ of "neurons" for different models. The multiequilibria conditions in this literature are normally tightly depending on the specific form of the dynamics and become inapplicable for the dynamics considered in this paper. The nonnegativity assumption on the adjacency matrix of the network yields two locally invariant regions, the positive and negative orthant, but outside these regions it is not possible to apply Brouwer fixed-point arguments, used to prove the existence of multiple equilibria in some of the papers cited above.

The main contribution of this paper is to investigate the multiequilibria problem using tools from Perron-Frobenius theory and geometrical considerations.

First, we prove that a necessary condition for the existence of equilibria outside $\mathbb{R}_{+}^{n}, \mathbb{R}_{-}^{n}$ is the existence of a second positive eigenvalue of the adjacency matrix. Equivalently, the value of the algebraic connectivity of the Laplacian of the system determines an interval of values to which the bifurcation parameter has to belong in order to guarantee the absence of mixed-signed equilibrium points. Numerically, it is possible to see that right beyond this value multiple equilibria appear. Second, we prove that for the case of identical nonlinearities the norm of each mixed-sign equilibrium 
point is less than the norm of the equilibrium point in the positive orthant, i.e., the norm of the positive equilibrium point represents the radius of the ball centered in the origin of $\mathbb{R}^{n}$ which must contain all the equilibria.

Most proofs of the results, here omitted for lack of space, appear in the extended journal paper [18].

\section{PRELIMINARIES}

\section{A. Nonnegative matrices and Perron-Frobenius}

The set of all $\lambda \in \mathbb{C}$ that are eigenvalues of $A \in \mathbb{R}^{n \times n}$ is called the spectrum of $A$ and is denoted by $\Lambda(A)$. The spectral radius of $A$ is the nonnegative real number $\rho(A)=$ $\max \{|\lambda|: \lambda \in \Lambda(A)\}$.

A matrix $B \in \mathbb{R}^{n \times n}$ is said to be similar to a matrix $A \in \mathbb{R}^{n \times n}$, abbreviated $B \sim A$, if there exists a nonsingular matrix $S \in \mathbb{R}^{n \times n}$ such that $B=S^{-1} A S$. If $A$ and $B$ are similar, then they have the same eigenvalues, counting multiplicity.

A matrix $A \in \mathbb{R}^{n \times n}$ is said to be reducible if either $n=$ 1 and $A=0$ or if $n \geq 2$, there is a permutation matrix $P \in \mathbb{R}^{n \times n}$ and there is some integer $r$ with $1 \leq r \leq n-$ 1 such that $P^{T} A P=\left[\begin{array}{cc}B & C \\ 0 & D\end{array}\right]$ where $B \in \mathbb{R}^{r \times r}, C \in$ $\mathbb{R}^{r \times(n-r)}, D \in \mathbb{R}^{(n-r) \times(n-r)}$. A matrix $A \in \mathbb{R}^{n \times n}$ is said to be irreducible if it is not reducible.

Theorem 1 (Perron-Frobenius, [19] Theorem 1.4) If $A \in \mathbb{R}^{n \times n}$ is irreducible and nonnegative then $\rho(A)$ is a real, positive, algebraically simple eigenvalue of $A$, of right (left) eigenvector $v>0(w>0)$. Furthermore $|\lambda|<\rho(A)$ for every eigenvalue $\lambda \in \Lambda(A)$ such that $\lambda \neq \rho(A)$.

Corollary 1 (Perron-Frobenius) If $A \in \mathbb{R}^{n \times n}$ is irreducible and nonnegative then either

$$
\rho(A)=\sum_{j=1}^{n} a_{i j}, \quad \forall i=1, \ldots, n
$$

or

$$
\min _{i}\left(\sum_{j=1}^{n} a_{i j}\right) \leq \rho(A) \leq \max _{i}\left(\sum_{j=1}^{n} a_{i j}\right) .
$$

\section{B. Symmetric, symmetrizable and congruent matrices}

Let $A \in \mathbb{R}^{n \times n}$ be symmetric. Then all the eigenvalues of $A$ are real and $S^{T} A S$ is symmetric for all $S \in \mathbb{R}^{n \times n}$.

A matrix $A \in \mathbb{R}^{n \times n}$ is (diagonally) symmetrizable if $D A$ is symmetric for some diagonal matrix $D$ with positive diagonal entries. The matrix $D A$ is called symmetrization of $A$ and the matrix $D$ is called symmetrizer of $A$. The eigenvalues of a symmetrizable matrix are real [20], [21], [22]. $A \in \mathbb{R}^{n \times n}$ is symmetrizable if and only if it is sign symmetric, i.e. $a_{i j}=a_{j i}=0$ or $a_{i j} \cdot a_{j i}>0, \forall i \neq j$, and $a_{i_{1}, i_{2}} a_{i_{2}, i_{3}} \ldots a_{i_{k}, i_{1}}=a_{i_{2}, i_{1}} a_{i_{3}, i_{2}} \ldots a_{i_{1}, i_{k}}$ for all $i_{i}, \ldots, i_{k}$.

A matrix $B \in \mathbb{R}^{n \times n}$ is said to be congruent to the matrix $A \in \mathbb{R}^{n \times n}$ if there exists a nonsingular matrix $S$ such that $B=S A S^{T}$. If $B$ is congruent to $A$, we say that $B$ can be obtained by a congruence transformation on $A$.
Theorem 2 (Ostrowski, Theorem 4.5.9 in [23]) Let $A, S \in \mathbb{R}^{n \times n}$ with $A$ symmetric and $S$ nonsingular. Let the eigenvalues of $A$ and $S S^{T}$ be arranged in nondecreasing order. For each $k=1, \ldots, n$ there exists a positive real number $\theta_{k}$ such that $\lambda_{1}\left(S S^{T}\right) \leq \theta_{k} \leq \lambda_{n}\left(S S^{T}\right)$ and $\lambda_{k}\left(S A S^{T}\right)=\theta_{k} \lambda_{k}(A)$.

\section{Cooperative system}

Consider the system

$$
\dot{x}=f(x), \quad x(0)=x_{0}
$$

where $f$ is a continuously differentiable function defined on a convex, open set $U \subseteq \mathbb{R}^{n}$. We write $x\left(t, x_{0}\right)$ for the forward solution of (3) with initial condition $x_{0} \in \mathbb{R}^{n}$ at $t=0$.

The set $\mathcal{K}$ given by $\mathcal{K}=\left\{x \in \mathbb{R}^{n}: k_{i} x_{i} \geq 0, k_{i} \in\right.$ $\{ \pm 1\}, i=1, \ldots, n\}$ is an orthant in $\mathbb{R}^{n} . \mathcal{K}$ is a cone in $\mathbb{R}^{n}$ and it generates a partial ordering " $\leq \mathcal{K}_{\mathcal{K}}$, i.e. $x \leq_{\mathcal{K}} y$ iff $y-x \in \mathcal{K}$. The subscript " $\mathcal{K}$ " will be dropped in case $\mathcal{K}=\mathbb{R}_{+}^{n}$, the nonnegative orthant.

System (3) is said to be type- $\mathcal{K}$ monotone [9] if whenever $\bar{x}$ and $\bar{y}$ lie in $U$ and if $\bar{x} \leq_{\mathcal{K}} \bar{y}$ then $x(t, \bar{x}) \leq_{\mathcal{K}} y(t, \bar{y})$ for all $t \geq 0$ for which both solutions are defined. In this case we say that the flow of (3) preserves the ordering $\leq \mathcal{K}$.

Lemma 1 ([9]) If $f \in C^{1}(U)$ where $U$ is open and convex in $\mathbb{R}^{n}$, then $x\left(t, x_{0}\right)$ preserves the partial ordering $\leq_{\mathcal{K}}$ for $t \geq 0$ if and only if $K \frac{\partial f}{\partial x}(x) K$ has nonnegative off-diagonal elements for every $x \in U$, where $K=\operatorname{diag}\left\{k_{1}, \ldots, k_{n}\right\}$, $k_{i} \in\{ \pm 1\}$, is the "signature" of the orthant $\mathcal{K}$.

If $\mathcal{K}=\mathbb{R}_{+}^{n}$ then we have the class of cooperative systems. System (3) is said to be cooperative in $U \subset \mathbb{R}^{n}$ if the differentiable vector field $f: \mathbb{R}^{n} \rightarrow \mathbb{R}^{n}$ is such that the Jacobian matrix $F(x):=\frac{\partial f}{\partial x}(x)$ is Metzler for all $x \in U$, that is $[F(x)]_{i j} \geq 0$ for all $i \neq j$.

\section{MULTIPLE EQUILIBRIA FOR NONLINEAR COOPERATIVE SYSTEMS}

The class of nonlinear systems considered in this work is the following [1], [16]

$$
\dot{x}=-\Delta x+\pi A \psi(x)
$$

where $x \in \mathbb{R}^{n}, \pi>0$ is a scalar parameter, $A=\left[a_{i j}\right]$ is the weighted adjacency matrix of the network and $\Delta=$ $\operatorname{diag}\left\{\delta_{1}, \ldots, \delta_{n}\right\}$ is the matrix of degradation terms $\delta_{i}$. The matrix $A$ is assumed to be nonnegative with null diagonal, irreducible and symmetrizable. Each $\delta_{i}$ value is defined as the $i$ th row sum of the matrix $A$, that is $\delta_{i}=\sum_{j} a_{i j}$, and from the irreducibility assumption on $A$ it follows that each $\delta_{i}$ is strictly positive, $\delta_{i}>0$. Moreover, the notation $\delta_{\min }=\min _{i}\left\{\delta_{i}\right\}$ and $\delta_{\max }=\max _{i}\left\{\delta_{i}\right\}$ is introduced.

The vector of functions $\psi(x)=\left[\psi_{1}\left(x_{1}\right) \ldots \psi_{n}\left(x_{n}\right)\right]^{T}$ is such that each $\psi_{i}\left(x_{i}\right): \mathbb{R} \rightarrow \mathbb{R}$ satisfies the following 
conditions

$$
\begin{gathered}
\psi_{i}\left(x_{i}\right)=-\psi_{i}\left(-x_{i}\right), \forall x_{i} \in \mathbb{R} \text { (odd) } \\
\frac{\partial \psi_{i}}{\partial x_{i}}\left(x_{i}\right)>0, \forall x_{i} \in \mathbb{R} \text { and } \frac{\partial \psi_{i}}{\partial x_{i}}(0)=1 \text { (monotone) } \\
\lim _{x_{i} \rightarrow \pm \infty} \psi_{i}\left(x_{i}\right)= \pm 1 \text { (saturated) }
\end{gathered}
$$

Typical choices for the nonlinear functions are a hyperbolic tangent function, a (modified) Michaelis-Menten function [3] or a (modified) Boltzmann function [5]. The last two are respectively $\psi_{i}\left(x_{i}\right)=\frac{x_{i}}{1+\left|x_{i}\right|}, x_{i} \in \mathbb{R}$ and $\psi_{i}\left(x_{i}\right)=\frac{1-e^{-2 x_{i}}}{1+e^{-2 x_{i}}}, x_{i} \in \mathbb{R}$. Revisited versions are here proposed in order to satisfy the conditions (A.1) $\div($ A.3) Additionally, if each nonlinear function $\psi_{i}\left(x_{i}\right)$ satisfies the following condition

$$
\begin{aligned}
& \psi_{i}\left(x_{i}\right) \text { strictly convex } \forall x_{i}<0 \text { and } \\
& \psi_{i}\left(x_{i}\right) \text { strictly concave } \forall x_{i}>0 \text { (sigmoidal) }
\end{aligned}
$$

then, from (A.2) and (A.4), $\left|\psi_{i}\left(x_{i}\right)\right|<\left|x_{i}\right|$ for all $i$ and $x_{i} \neq 0$. The examples mentioned above (hyperbolic tangent, Michaelis-Menten and Boltzmann functions) satisfy (A.4).

From these assumptions, it follows that the Jacobian matrix of (4), given by $-\Delta+\pi A \frac{\partial \psi}{\partial x}(x)$, is Metzler. Therefore, the system (4) is cooperative.

It is convenient to introduce the matrix $H:=\pi \Delta^{-1} A$. Observe that it satisfies some useful properties:

- It is nonnegative and irreducible, so Theorem 1 applies.

- All the row sums are equal to $\pi$, that is $H \mathbb{1}_{n}=\pi \mathbb{1}_{n}$. It follows that $\left(\pi, \mathbb{1}_{n}\right)$ is an eigenpair of the matrix $H$. Then, by Theorem $1, \rho(H)=\pi$.

- As the matrix $A$ is symmetrizable, i.e. there exist a positive diagonal matrix $D$ and a symmetric matrix $S_{A}$ such that $A=D S_{A}$, also $H$ is symmetrizable since there exists a positive definite diagonal matrix $D_{H}$, given by $D_{H}:=D^{-1} \Delta$, such that $D_{H} H$ is symmetric. Then $H$ has real eigenvalues.

In the following analysis the matrix $H$ is used to rewrite the system (4) in a different form

$$
\dot{x}=\Delta[-x+H \psi(x)], \quad x \in \mathbb{R}^{n} .
$$

We will see below that the existence (and the stability) of multiple equilibria is strictly related to the structure of the spectrum of the matrix $H$.

\section{A. Existence of multiple equilibria}

Consider the system (4) (or (5)) where each nonlinear function $\psi_{i}\left(x_{i}\right)$ satisfies the properties (A.1) $\div$ (A.4). Let us start by recalling what is known for this system when we vary the parameter $\pi$. By construction, the origin is always an equilibrium point for (4) (or (5)). When $\pi<1, x=0$ is the only equilibrium point, it is globally asymptotically stable and locally exponentially stable. This follows from diagonal dominance, and can be easily shown by a Lyapunov argument, see [1], [16], [17], [24]. At $\pi=1$ the system undergoes a pitchfork bifurcation, the origin becomes a saddle point and two more equilibria emerge, $x^{+} \in \mathbb{R}_{+}^{n}$ and $x^{-} \in \mathbb{R}_{-}^{n}$ [1]. It follows from the analysis of [16] that, for all $\pi>1, x=0$ is an unstable equilibrium point, while both $x^{+}$and $x^{-}$are locally asymptotically stable with domain of attraction given by the entire orthant. $\mathbb{R}_{+}^{n}$ (resp. $\mathbb{R}_{-}^{n}$ ) is in fact invariant for the system (4). What happens outside these two orthants is however unknown. When $\pi>1$ and $\pi-1$ sufficiently small, the behavior of the system (4) outside $\mathbb{R}_{+}^{n}$ and $\mathbb{R}_{-}^{n}$ has been discussed in [1]. Only the three equilibrium points mentioned above are possible, two locally stable [16] and the origin as a saddle point. However, when $\pi>1$ grows, the bifurcation analysis of [1] does not hold anymore.

Our task is therefore to investigate the behavior of the system (4) when $\pi>1$ grows and $x \in \mathbb{R}^{n}$ (case not described by [16] and [1]). In particular, we would like to understand for what interval $(1, \bar{\pi})$ of the bifurcation parameter $\pi$ extra equilibria not contained in $\mathbb{R}_{+}^{n} / \mathbb{R}_{-}^{n}$ cannot appear, and what happens for $\pi>\bar{\pi}$.

The following theorem introduces a necessary condition that has to be verified in order to have an equilibrium point $\bar{x}$ in a generic orthant $\mathcal{K} \neq \mathbb{R}_{+}^{n}, \mathbb{R}_{-}^{n}$ for the system (4).

Theorem 3 Consider the system (4), where each nonlinear function $\psi_{i}\left(x_{i}\right)$ satisfies the properties (A.1) $\div$ (A.4). If the system admits an equilibrium point $\bar{x} \in \mathcal{K}$, where $\mathcal{K}$ is an orthant in $\mathbb{R}^{n}$ and $\mathcal{K} \neq \mathbb{R}_{+}^{n}, \mathbb{R}_{-}^{n}$, then $\exists \lambda(A) \in \Lambda(A)$ such that $\lambda(A)>0$ and $\lambda(A) \neq \rho(A)$ for which $\pi \lambda(A)>\delta_{\text {min }}$.

In order to prove the theorem we need the following auxiliary lemma.

Lemma 2 Consider the system (5) where each nonlinear function $\psi_{i}\left(x_{i}\right)$ satisfies the properties (A.1) $\div$ (A.4). If the system admits an equilibrium point $\bar{x} \in \mathcal{K}$, where $\mathcal{K}$ is an orthant in $\mathbb{R}^{n}$ and $\mathcal{K} \neq \mathbb{R}_{+}^{n}, \mathbb{R}_{-}^{n}$, then $\exists \lambda(H) \in \Lambda(H)$ such that $\lambda(H)>1$ and $\lambda(H) \neq \rho(H)$.

Proof. Let $\bar{x} \in \mathcal{K}$ be an equilibrium point for (5). Because $\Delta$ is diagonal and positive definite, from (5) it follows

$$
\bar{x}=H \psi(\bar{x}) .
$$

First notice that if $\bar{x} \in \mathcal{K}$ also $\psi(\bar{x}) \in \mathcal{K}$ because, from (A.1) and (A.2), $\psi_{i}\left(x_{i}\right)$ keeps the same sign of $x_{i}$ for all $i=1, \ldots, n$. Introduce the diagonal matrix $M(\bar{x})=$ $\operatorname{diag}\left\{m_{1}\left(\bar{x}_{1}\right), \ldots, m_{n}\left(\bar{x}_{n}\right)\right\}$ where each element is given by

$$
m_{i}\left(\bar{x}_{i}\right)=\frac{\psi_{i}\left(\bar{x}_{i}\right)}{\bar{x}_{i}}, \quad i=1, \ldots, n .
$$

Since $\bar{x}>_{\mathcal{K}} 0$, the ratio is well-posed. The dependence of $M(\bar{x})$ from $\bar{x}$ will be omitted from now on. From (A.2) and (A.4) one gets $\left|\psi_{i}\left(x_{i}\right)\right|<\left|x_{i}\right| \forall i, x_{i} \neq 0$, which leads to $m_{i}=\frac{\psi_{i}\left(\bar{x}_{i}\right)}{\bar{x}_{i}} \in(0,1)$ for all $i$. Then $0<\operatorname{diag}(M)<\mathbb{1}_{n}$.

Knowing that $\psi(\bar{x})=M \bar{x}$, the matrix $M$ can be used to rewrite (6); moreover, applying the change of coordinates $\bar{z}=M^{1 / 2} \bar{x}$ leads to

$$
\bar{z}=M^{1 / 2} H M^{1 / 2} \bar{z} .
$$


Equation (7) represents the eigenvalue equation for the matrix $M^{1 / 2} H M^{1 / 2}$, that is $(1, \bar{z})$ is an eigenpair of $M^{1 / 2} H M^{1 / 2}$. Furthermore $\bar{z} \in \mathcal{K}$ since, for each $i$, $\bar{z}_{i}=\sqrt{m_{i}} \bar{x}_{i}$ and $m_{i}$ is strictly positive. Observe that $M^{1 / 2} H M^{1 / 2}$ is nonnegative and irreducible and let its eigenvalues be arranged in a nondecreasing order. Theorem 1 states that $\lambda_{n}\left(M^{1 / 2} H M^{1 / 2}\right)=\rho\left(M^{1 / 2} H M^{1 / 2}\right)$ is real and positive and that its associated eigenvector is real and in the positive orthant of $\mathbb{R}^{n}$. Then $\rho\left(M^{1 / 2} H M^{1 / 2}\right)>1$, since the eigenvector associated to 1 is $\bar{z} \in \mathcal{K} \neq \mathbb{R}_{+}^{n}, \mathbb{R}_{-}^{n}$. To prove that $\exists \lambda(H) \in \Lambda(H)$ such that $\lambda(H)>1$ we proceed in steps.

- Since the matrix $A$ is symmetrizable, let $A=D_{A} S_{A}$ where $D_{A}$ is a diagonal matrix with positive diagonal entries and $S_{A}$ is a symmetric matrix. The matrix $S_{A}$ is still irreducible, nonnegative and with null diagonal.

- The matrix $H$, which can be written as $H=$ $\pi \Delta^{-1} D_{A} S_{A}$, is symmetrizable. Define the matrix $D_{H}:=\pi \Delta^{-1} D_{A}$, diagonal with positive diagonal entries. Then $S_{A}=D_{H}^{-1} H$ is the symmetrization of $H$ while $D_{H}^{-1}$ is the symmetrizer of $H$.

- Consider the matrix $\tilde{H}$ defined as

$$
\tilde{H}:=D_{H}^{-1 / 2} H D_{H}^{1 / 2}=D_{H}^{1 / 2} S_{A} D_{H}^{1 / 2} .
$$

By construction it is symmetric, nonnegative, irreducible and similar to $H$. Because $H$ and $\tilde{H}$ have the same eigenvalues, it is just necessary to prove that $\exists \lambda(\tilde{H}) \in \Lambda(\tilde{H})$ such that $\lambda(\tilde{H})>1$ and $\lambda(\tilde{H}) \neq$ $\rho(\tilde{H})$.

- The matrices $M^{1 / 2} H M^{1 / 2}$ and $M^{1 / 2} \tilde{H} M^{1 / 2}$ are similar. Indeed

$$
\begin{aligned}
M^{1 / 2} H M^{1 / 2} & =D_{H}^{1 / 2}\left[M^{1 / 2} \tilde{H} M^{1 / 2}\right] D_{H}^{-1 / 2} \\
& \sim M^{1 / 2} \tilde{H} M^{1 / 2} .
\end{aligned}
$$

Then they have the same eigenvalues and in particular, from equation (7), it follows that $\left(1, D_{H}^{-1 / 2} \bar{z}\right)$ is an eigenpair of the matrix $M^{1 / 2} \tilde{H} M^{1 / 2}$. Since $D_{H}^{-1 / 2} \bar{z} \in \mathcal{K}$, it follows that $\exists k \neq n$ such that $\lambda_{k}\left(M^{1 / 2} \tilde{H} M^{1 / 2}\right)=1$.

- The matrix $M^{1 / 2} \tilde{H} M^{1 / 2}$ is symmetric and $M^{1 / 2}$ is nonsingular so it is possible to apply Theorem 2 . There exists a positive real number $\theta_{k}$ such that

$$
\lambda_{k}\left(M^{1 / 2} \tilde{H} M^{1 / 2}\right)=\theta_{k} \lambda_{k}(\tilde{H})
$$

and

$$
\lambda_{1}\left(M^{1 / 2}\left(M^{1 / 2}\right)^{T}\right) \leq \theta_{k} \leq \lambda_{n}\left(M^{1 / 2}\left(M^{1 / 2}\right)^{T}\right)
$$

where $\lambda_{1}(M)=m_{\min }$ and $\lambda_{n}(M)=m_{\max }$. Then $\theta_{k} \leq m_{\max }<1$. Since $k \neq n$ is the index for which $\lambda_{k}\left(M^{1 / 2} \tilde{H} M^{1 / 2}\right)=1$, it follows

$$
1=\lambda_{k}\left(M^{1 / 2} \tilde{H} M^{1 / 2}\right)=\theta_{k} \lambda_{k}(\tilde{H})<\lambda_{k}(\tilde{H})
$$

Because $k \neq n$, this implies the existence of an eigenvalue $\lambda(\tilde{H}) \in \Lambda(\tilde{H})$ such that $\lambda(\tilde{H})>1$ and $\lambda(\tilde{H}) \neq \rho(\tilde{H})$. Consequently, since $H$ and $\tilde{H}$ are similar, there exists $\lambda(H) \in \Lambda(H)$ such that $\lambda(H)>1$ and $\lambda(H) \neq \rho(H)$.

Proof. [Proof of Theorem 3] Let the symmetrizable matrix $A$ be written as $A=D S_{A}$, where $D$ is a diagonal matrix with positive diagonal entries and $S_{A}$ is a symmetric matrix. Then $H=\pi \Delta^{-1} D S_{A}$. Define a new matrix

$$
\tilde{H}:=\Delta^{1 / 2} D^{-1 / 2} H D^{1 / 2} \Delta^{-1 / 2}=\pi \Delta^{-1 / 2} \tilde{A} \Delta^{-1 / 2}
$$

where $\tilde{A}$ is defined as $\tilde{A}:=D^{-1 / 2} A D^{1 / 2}=D^{1 / 2} S_{A} D^{1 / 2}$. By construction, $\tilde{H}$ is symmetric, similar to $H$ and congruent to $\tilde{A}$, while $\tilde{A}$ is symmetric, similar to $A$ and congruent to $S_{A}$. Because $\tilde{H}$ and $\tilde{A}$ are both symmetric, it is possible to apply Theorem 2. To simplify the notation, let $\tilde{S}:=$ $\sqrt{\pi} \Delta^{-1 / 2}$ and $\tilde{H}=\tilde{S} \tilde{A} \tilde{S}^{T}$, and the eigenvalues be arranged in a nondecreasing order. Therefore, there exists a positive real number $\theta_{k}$ such that the following conditions hold

$$
\begin{gathered}
\lambda_{k}(\tilde{H})=\theta_{k} \lambda_{k}(\tilde{A}) \\
\lambda_{1}\left(\tilde{S} \tilde{S}^{T}\right) \leq \theta_{k} \leq \lambda_{n}\left(\tilde{S} \tilde{S}^{T}\right)
\end{gathered}
$$

From Lemma $2, \exists k \neq n$ such that $\lambda_{k}(H)>1$. It follows, by similarity, that $\lambda_{k}(\tilde{H})>1$. Then the condition (8) where $\theta_{k}>0$ and $k \neq n$, yields $\lambda_{k}(\tilde{A})>0$ and $\lambda_{k}(\tilde{A}) \neq \rho(\tilde{A})$. Moreover, since $\tilde{S} \tilde{S}^{T}=\pi \Delta^{-1}$, then $\lambda_{1}\left(\tilde{S} \tilde{S}^{T}\right)=\frac{\pi}{\delta_{\max }}$ and $\lambda_{n}\left(\tilde{S} \tilde{S}^{T}\right)=\frac{\pi}{\delta_{\min }}$. From (9), (8) and the result of Lemma 2, it follows that $1<\frac{\pi}{\delta_{\min }} \lambda_{k}(\tilde{A})$, i.e., $\pi \lambda_{k}(\tilde{A})>\delta_{\min }$. But $\tilde{A}$ and $A$ are similar, that is, they have the same eigenvalues. Then $\pi \lambda_{k}(A)>\delta_{\min }$, which concludes the proof.

Remark 1 Observe that if $\bar{x} \in \mathbb{R}^{n}$ is any equilibrium point for the system (4) (or (5)), also $-\bar{x}$ is an equilibrium point as well.

Remark 2 The necessary condition given by Theorem 3 implies that in order to have an equilibrium point $\bar{x} \in \mathcal{K}$ for the system (4), it must be $n>3$. Indeed it is easy to show that it is impossible for $A$ to have a second positive eigenvalue which differs from the Perron-Frobenius eigenvalue if $n \leq 3$.

\section{B. Geometric interpretation}

It is useful to give a geometric interpretation of the necessary conditions of Theorem 3 and Lemma 2. Let us consider the matrix $H$, for which such an interpretation is easier. Denote $H_{1}=\Delta^{-1} A$ the value of $H$ when $\pi=1$, so that $H=\pi H_{1}$. For $H_{1}, \rho\left(H_{1}\right)=1$ and (1) holds: $\sum_{j} h_{1, i j}=\rho\left(H_{1}\right)=1 \forall i$. This means that the Laplacian $L_{1}=I-H_{1}$ has all identical Geršgorin disks, all centered at 1 and passing through the origin:

$$
\left\{s \in \mathbb{C} \text { s.t. }|s-1| \leqslant \sum_{j=1}^{n} \frac{a_{i j}}{\delta_{i}}=1\right\} .
$$

From Geršgorin Theorem [23], the eigenvalues of $L_{1}$ are located in the union of the $n$ disks. By construction, the 
least eigenvalue $\lambda_{1}\left(L_{1}\right)$ is the origin and corresponds to $\rho\left(H_{1}\right)$. The other eigenvalues of $L_{1}$ are strictly inside the disks because of irreducibility and positive semidefiniteness of $L_{1}$. When $\pi>1$, the Geršgorin disks are still all centered in 1 but have radius $\pi$, and all the eigenvalues of $H=\pi H_{1}$ are increased in modulus. If $\lambda_{n-1}\left(H_{1}\right)$ is the second largest positive eigenvalue of $H_{1}$, i.e., the second leftmost eigenvalue of $L_{1}$ (also called algebraic connectivity [25]), then the necessary condition of Lemma 2 corresponds to requiring that the value of $\pi$ is big enough so as to allow $1-\pi \lambda_{n-1}\left(H_{1}\right)$ to be in the left half of the complex plane.For this to happen the radius of all the Geršgorin disks of $I-H, \pi$, has to be $>\frac{1}{\lambda_{n-1}\left(H_{1}\right)}$. The previous arguments are summarized in the following proposition.

Proposition 1 The range $(1, \bar{\pi})$ of values of $\pi$ for which no extra equilibrium of the system (5) (other than $0, x^{+}$and $x^{-}$) can appear is determined by the algebraic connectivity of $L_{1}=I-H_{1}$.

If instead we look at system (4), and at the Laplacian $L=\Delta-A$, then the Geršgorin disks are centered at $\delta_{i}$ and have different radii, equal to $\delta_{i}$, when $\pi=1$. However, this cannot be straightforwardly reformulated in terms of $\rho(A)$, as (2) (instead of (1)) now holds: $\delta_{\min } \leq \rho(A) \leq \delta_{\max }$. When exploring the values $\pi>1$, then the Geršgorin disks of $\Delta-\pi A$ are contained one in the other, according to the corresponding $\delta_{i}$, and all have non empty intersection with the left half of $\mathbb{C}$.

Let $\pi>1$ and $\tilde{L}$ be defined as $\tilde{L}:=\Delta-\pi A$. In the following discussion we analyze $\tilde{L}$, considering the different necessary conditions described by Lemma 2 and Theorem 3 (proofs are omitted for lack of space).

Proposition 2 The condition introduced by Lemma 2 represents the necessary and sufficient condition in order for $\tilde{L}$ to have a second leftmost eigenvalue which is negative.

Proposition 3 The condition introduced by Theorem 3 represents a necessary condition for $\tilde{L}$ to have a second leftmost eigenvalue in the left half of the complex plane.

\section{Stability properties of multiple equilibria}

Theorem 4 Suppose the system (4) admits an equilibrium point $\bar{x} \in \mathcal{K}$, where $\mathcal{K}$ is an orthant in $\mathbb{R}^{n}$ and $\mathcal{K} \neq \mathbb{R}_{+}^{n}, \mathbb{R}_{-}^{n}$. If

$$
\pi \max _{j}\left\{\frac{\partial \psi_{j}}{\partial x_{j}}\left(\bar{x}_{j}\right)\right\}<1
$$

then $\bar{x}$ is locally asymptotically stable. Instead, if

$$
\pi \min _{j}\left\{\frac{\partial \psi_{j}}{\partial x_{j}}\left(\bar{x}_{j}\right)\right\}>1
$$

then the equilibrium point $\bar{x}$ is unstable.

The proof, omitted for lack of space, is available in [18].

\section{LOCATION OF THE MIXED-SIGN EQUILIBRIA}

In this Section we restrict our analysis to the special case of all identical $\psi_{i}\left(x_{i}\right)$. In this case the equilibrium point in the positive orthant has all identical components as shown in the following lemma, whose proof is omitted for lack of space.

Lemma 3 Consider the system (5) where each $\psi_{i}\left(x_{i}\right)$ satisfies the properties (A.1) $\div$ (A.4) and $\psi_{i}(\xi)=\psi_{j}(\xi) \forall i, j$ and $\forall \xi \in \mathbb{R}$. When $\pi>1$ the positive equilibrium point $x^{+} \in \mathbb{R}_{+}^{n}$ is such that $\frac{\psi_{i}\left(x_{i}^{+}\right)}{x_{i}^{+}}=\frac{1}{\pi}$. Furthermore $x^{+}$is the Perron-Frobenius (right) eigenvector of $H$.

A more important consequence is that for each value of $\pi$ the positive equilibrium point $x^{+}$provides an upper bound on the norm that any mixed-sign equilibrium $\bar{x}$ can assume.

Theorem 5 Consider the system (4), where each nonlinear function $\psi_{i}\left(x_{i}\right)$ satisfies the properties (A.1) $\div$ (A.4) and $\psi_{i}(\xi)=\psi_{j}(\xi) \forall i, j$ and $\forall \xi \in \mathbb{R}$. If the system admits an equilibrium point $\bar{x} \in \mathcal{K}$, where $\mathcal{K}$ is an orthant in $\mathbb{R}^{n}$ and $\mathcal{K} \neq \mathbb{R}_{+}^{n}, \mathbb{R}_{-}^{n}$, then $\|\bar{x}\| \leq\left\|x^{+}\right\|$.

The proof is available in [18].

\section{EXAMPLES}

In this Section we first look at the special case of adjacency matrices of $\{0,1\}$ which are full or nearly full. Then we investigate the width of the interval $(1, \bar{\pi})$ in which no equilibria of (4) other than $0, x^{+}$and $x^{-}$appear, when networks with weighted adjacency matrix are considered.

\section{A. Full or nearly full $\{0,1\}$ adjacency matrices}

Consider the case of an irreducible symmetric nonnegative matrix $A$ whose nondiagonal elements can assume values only in $\{0,1\}$.

Proposition 4 Let the matrix $A=\left[a_{i j}\right]$ be such that $a_{i j}=1$ if $i \neq j$ and $a_{i j}=0$ if $i=j$, i.e. $A$ is full. Then the PerronFrobenius eigenvalue is the only positive eigenvalue of $A$ and the necessary condition given by Theorem 3 does not hold.

Proof. The matrix can be rewritten as $A=E-I$, where $E=\mathbb{1}_{n} \mathbb{1}_{n}^{T}$. The matrix $E$ has rank 1 , that is, it has $n-1$ zero eigenvalues; since it is also positive and irreducible, its Perron-Frobenius eigenvalue is given by $\rho(E)=n$. This implies that $\rho(A)=n-1$, while the other $n-1$ eigenvalues are all placed in -1 .

Proposition 5 Given $\bar{i}, \bar{j} \in\{1, \ldots, n\}, \bar{i} \neq \bar{j}$, let the matrix $A$ be such that

$$
\left\{\begin{array}{l}
a_{i j}=1, \forall i, j \in\{1, \ldots, n\}, i \neq \bar{i}, j \neq \bar{j}, i \neq j \\
a_{i j}=0, \text { otherwise }
\end{array}\right.
$$

i.e. each non diagonal element of $A$ is one except for the two elements $a_{\bar{i}, \bar{j}}$ and $a_{\bar{j}, \bar{i}}$, and each diagonal element is zero. Then the Perron-Frobenius eigenvalue is the only strictly 
positive eigenvalue of $A$ and the necessary condition given by Theorem 3 does not hold.

Proof. The matrix $A+I$ has rank 3 , that is it has $n-3$ zero eigenvalues. Since it is irreducible and nonnegative $\rho(A+I)$ is the Perron-Frobenius eigenvalue and by construction $1 \in$ $\Lambda(A+I)$. Since its trace is $n$, it follows that the last nonzero eigenvalue of $A+I$ is given by $\lambda_{1}(A+I)=n-\rho(A+I)-1$. It is nonpositive since $\rho(A+I) \in[n-1, n]$. As a consequence the matrix $A$ has $n-3$ eigenvalues in -1 , Perron-Frobenius eigenvalue $\rho(A)=\rho(A+I)-1$, one zero eigenvalue and one eigenvalue in $\lambda_{1}(A+I)-1<0$.

If instead the nondiagonal zero elements of the matrix $A$ are more than 2 , then the necessary condition of Theorem 3 may be satisfied.

\section{B. Weighted adjacency matrices}

Example 1 The necessary condition of Theorem 3 can be used to estimate the range of values of the parameter $\pi$ for which no mixed-sign equilibria of (4) can appear. It is actually convenient to consider the equivalent system (5) and the condition of Lemma 2. Consider networks of size $n=20$ (Erdős-Rényi graphs with edge probability $p=0.1$, and edges drawn from a uniform distribution). In the inset of Figure 1, it can be observed that when $\pi$ becomes bigger than 1, the second positive eigenvalue of $H$ passes 1 very quickly (values of 200 different realizations of $A$ are shown in the figure), meaning that the interval $(1, \bar{\pi})$ in which the system can have only $0, x^{+}$and $x^{-}$as equilibria is fairly narrow. This reinforces our intuition that the necessary condition of Lemma 2 could be also sufficient. When the result is mapped into the system (4) and the necessary condition of Theorem 3, then we have the distribution of value in the large panel of Fig. 1. What can be observed in this case is that the condition $\pi>\frac{\delta_{\min }}{\lambda_{n-1}(A)}$ is never tight $\left(\frac{\delta_{\min }}{\lambda_{n-1}(A)}\right.$ is never bigger than 0.5 , while $\pi>1$ ). This is due to the gap appearing in this case between $\delta_{\min }$ and $\delta_{\max }$.

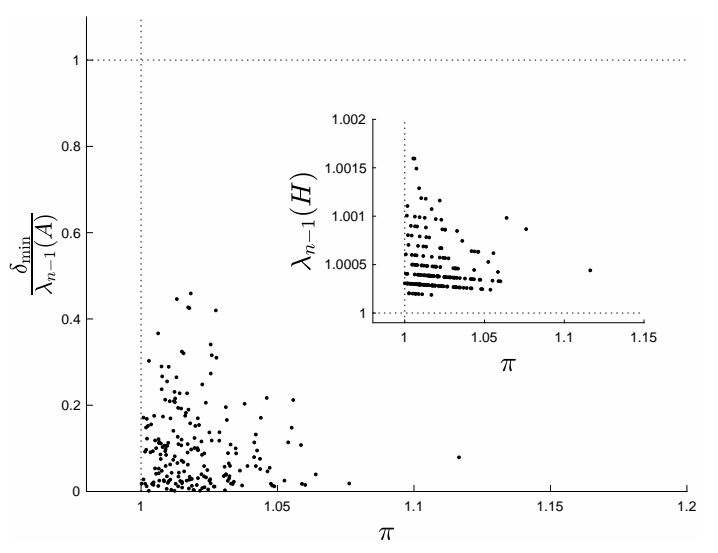

Fig. 1. Example 1. Range of values $(1, \bar{\pi})$ of $\pi$ for which no extra equilibria appear. Large panel: the values of $\bar{\pi}$ at which equilibria $\bar{x}$ start to appear are shown, versus $\frac{\delta_{\min }}{\lambda_{n-1}(A)}$ for 200 different realizations of $A$ of size $n=20$. $\bar{\pi}$ is often very close to 1 . Inset: same values of $\bar{\pi}$ but now compared with $\lambda_{n-1}(H)$.

\section{REFERENCES}

[1] A. Franci, V. Srivastava, and N. E. Leonard, "A Realization Theory for Bio-inspired Collective Decision-Making," arXiv preprint, 2015.

[2] C. Altafini and G. Lini, "Predictable dynamics of opinion forming for networks with antagonistic interactions," IEEE Transactions on Automatic Control, vol. 60, no. 2, pp. 342-357, 2015.

[3] C. Altafini, "Dynamics of opinion forming in Structurally Balanced Social Networks," PLoS ONE, vol. 7, no. 6, pp. 1-9, 2012.

[4] C. Li, L. Chen, and K. Aihara, "Stability of genetic networks with SUM regulatory logic: Lur'e system and LMI approach," IEEE Transactions on Circuits and Systems I: Regular Papers, vol. 53, no. 11, pp. 2451-2458, 2006.

[5] S. Haykin, Neural networks: a Comprehensive Foundation, 2nd ed. Prentice Hall PTR, 1998.

[6] J. J. Hopfield, "Neurons with graded response have collective computational properties like those of two-state neurons." Proceedings of the National Academy of Sciences, vol. 81, no. 10, pp. 3088-3092, 1984.

[7] E. Kaszkurewicz and A. Bhaya, Matrix diagonal stability in systems and computation. Boston: Birkhäuser, 2000.

[8] M. W. Hirsch and H. Smith, "Monotone Dynamical Systems," in Handbook of Differential Equations: Ordinary Differential Equations, 2006, vol. 2, ch. 4, pp. 239-357.

[9] H. L. Smith, "Systems of Ordinary Differential Equations Which Generate an Order Preserving Flow. A Survey of Results," SIAM Review, vol. 30, no. 1, pp. 87-113, 1988.

[10] M. A. Cohen and S. Grossberg, Artificial Neural Networks: Theoretical Concepts, V. Vemuri, Ed. IEEE Computer Society Press, 1988.

[11] C.-Y. Cheng, K.-H. Lin, and C.-W. Shih, "Multistability in Recurrent Neural Networks," SIAM Journal on Applied Mathematics, vol. 66, no. 4, pp. 1301-1320, 2006.

[12] C. Y. Cheng, K. H. Lin, and C. W. Shih, "Multistability and convergence in delayed neural networks," Physica D: Nonlinear Phenomena, vol. 225 , no. 1 , pp. 61-74, 2007.

[13] Z. Zeng and W. X. Zheng, "Multistability of neural networks with time-varying delays and concave-convex characteristics," IEEE Transactions on Neural Networks and Learning Systems, vol. 23, no. 2, pp. 293-305, 2012.

[14] W. Lu, L. Wang, and T. Chen, "On attracting basins of multiple equilibria of a class of cellular neural networks," IEEE Transactions on Neural Networks, vol. 22, no. 3, pp. 381-394, 2011.

[15] H. Zhang, Z. Wang, and D. Liu, "A comprehensive review of stability analysis of continuous-time recurrent neural networks," IEEE Transactions on Neural Networks and Learning Systems, vol. 25, no. 7, pp. 1229-1262, 2014.

[16] P. Ugo Abara, F. Ticozzi, and C. Altafini, "Spectral conditions for stability and stabilization of positive equilibria for a class of nonlinear cooperative systems," IEEE Transactions on Automatic Control, to appear, 2017.

[17] _ "Existence, uniqueness and stability properties of positive equilibria for a class of nonlinear cooperative systems," in 54th IEEE Conference on Decision and Control, Osaka, Japan, 2015.

[18] A. Fontan and C. Altafini, "Multiequilibria analysis for a class of collective decision-making networked systems," IEEE Transactions on Control of Network Systems, conditionally accepted, 2017.

[19] A. Berman and R. J. Plemmons, Nonnegative Matrices in the Mathematical Sciences, ser. Classic in applied mathematics. Society for Industrial and Applied Mathematics, 1994.

[20] E. Kaszkurewicz and A. Bhaya, Matrix Diagonal Stability in Systems and Computation. Boston: Birkhäuser, 2000.

[21] E. S. Dias, D. Castonguay, and M. C. Dourado, "Algorithms and Properties for Positive Symmetrizable Matrices," TEMA (São Carlos), vol. 17, no. 2, pp. 187 - 198, 2016.

[22] S. Camiz and S. Stefani, Matrices and Graphs. World Scientific, 1996.

[23] R. A. Horn and C. R. Johnson, Matrix Analysis, 1st ed. Cambridge University Press, 1990.

[24] V. S. Bokharaie, O. Mason, and F. Wirth, "Stability and positivity of equilibria for subhomogeneous cooperative systems," Nonlinear Analysis: Theory, Methods \& Applications, vol. 74, no. 17, pp. 6416 - 6426, 2011.

[25] R. Olfati-Saber and R. Murray, "Consensus problems in networks of agents with switching topology and time-delays," Automatic Control, IEEE Transactions on, vol. 49, no. 9, pp. 1520 - 1533, sept. 2004. 